\title{
Prevenção da reincidência de gravidez em adolescentes: efeitos de um Programa de Planejamento Familiar*
}

\author{
The effectiveness of an educational family planning program in preventing the recurrence of teen \\ pregnancy
}

Prevención de la reincidencia del embarazo en adolescentes: efectos de un programa de planificación familiar

\author{
Luciana Mendes Berlofi ${ }^{1}$, Eloisa Luci Cardoso Alkmin ${ }^{1}$, Márcia Barbieri ${ }^{2}$, \\ Cristina Aparecida Falbo Guazzelli ${ }^{3}$, Fabio Fernando de Araújo ${ }^{4}$
}

\begin{abstract}
RESUMO
Objetivo: avaliar os efeitos de um programa educativo e assistencial frente a reincidência de gestação em adolescentes. Métodos: estudo descritivo e retrospectivo, realizado no setor de Planejamento Familiar da Universidade Federal de São Paulo a partir de dados coletados em 264 prontuários de adolescentes que tinham como antecedente ao menos uma gravidez anterior a matrícula. Resultados: os dados revelam que a menarca ocorreu, em média, aos 12,2 anos, a sexarca aos 15 e a primeira gravidez um ano após. Ao se matricularem no programa $73,5 \%$ possuíam uma gestação e $2 \%$ duas. O condom e os hormonais foram os contraceptivos mais utilizados. A reincidência de gravidez foi observada em 4,9\% das adolescentes. Conclusões: Os dados encontrados reforçam a importância do oferecimento de programas de planejamento familiar, que englobem a educação e a assistência com oferta do método contraceptivo, voltados a adolescentes.

Descritores: Gravidez; Adolescente; Gravidez na adolescência; Anticoncepção; Serviços de planejamento familiar
\end{abstract}

\begin{abstract}
Objective: to evaluate the effectiveness of an educational family planning program in preventing the recurrence of teen pregnancy. Methods: this was a retrospective study conducted in the department of Family Planning of the Federal University of São Paulo. The sample consisted of 264 patients who had at least one pregnancy. Results: participants reported an average age of menarche at 12.2 years old. Their first sexual intercourse occurred at 15 years old and their age at the first pregnancy was 16 years old. In addition, $73.5 \%$ of the participants reported more than one pregnancy. Following educational family planning program, use of condoms, and birth control pills were the most used contraceptives methods; $4.9 \%$ of teens had a recurrence of pregnancy. Conclusion: the findings of this study support the importance and effectiveness of an educational family planning program in preventing recurrence of teen pregnancy.
\end{abstract}

Keywords: Pregnancy; Adolescence; Pregnancy in adolescence; Contraception; Family planning services

\section{RESUMEN}

Objetivo: evaluar los efectos de un programa educativo y asistencial frente a la reincidencia de embarazo en adolescentes. Métodos: estudio descriptivo y retrospectivo, realizado en el sector de Planificación Familiar de la Universidad Federal de Sao Paulo a partir de datos recolectados en 264 historias clínicas de adolescentes que tenían como antecedente al menos un embarazo anterior a la matrícula. Resultados: los datos revelan que la menarquia ocurrió, en promedio, a los 12,2 años, la primera relación a los 15 y el primer embarazo un año después. $\mathrm{Al}$ matricularse en el programa el 73,5\% poseían una gestación y el 2\% dos. El condón y las hormonas fueron los anticonceptivos más utilizados. La reincidencia de embarazo fue observada en el 4,9\% de las adolescentes. Conclusiones: los datos encontrados refuerzan la importancia del ofrecimiento de programas de planificación familiar que abarquen la educación y la asistencia con la oferta de métodos anticonceptivos volcados a las adolescentes.

Descriptors: Embarazo; Adolescente; Embarazo en adolescencia; Anticoncepción; Servicios de planificación familiar

\footnotetext{
* Trabalbo realizado no Setor de Planejamento Familiar da Universidade Federal de São Paulo - UNIFESP - São Paulo (SP), Brasil.

${ }^{1}$ Enfermeira graduada pela Universidade Federal de São Paulo - UNIFESP - São Paulo (SP), Brasil.

2 Professora Adjunto do Departamento de Enfermagem da Universidade Federal de São Paulo - UNIFESP - São Paulo (SP), Brasil.

${ }^{3}$ Professora Adjunto do Departamento de Obstetrícia da Universidade Federal de São Paulo - UNIFESP - São Paulo (SP), Brasil.

${ }^{4}$ Professor Adjunto do Departamento de Ginecologia da Universidade Federal de São Paulo-- - UNIFESP - São Paulo (SP), Brasil.
} 


\section{INTRODUÇÃO}

Considera-se adolescência o período de 10 a 19 anos de idade, sendo compreendida como o período de vida a partir do qual surgem as características sexuais secundárias e se desenvolvem processos psicológicos e padrões de identificação que evoluem da fase infantil para a adulta, entre eles a transição de um estado de dependência para outro de relativa autonomia ${ }^{(1)}$.

Pesquisa realizada no país em 1996 revelou que 18\% das adolescentes brasileiras entre 15 e 19 anos já tiveram pelo menos um filho ${ }^{(2)}$. Anualmente, cerca de um milhão de jovens de 10 a 19 anos de idade se tornam mães.

Apesar de muito freqüente, nem sempre a gravidez na adolescência é um evento único, fortuito, que "escapou" ao controle, visto que, para algumas jovens, isto acaba se repetindo. Entretanto, pior que uma gestação na adolescência é sua repetição, que pressupõe problemas como o pequeno intervalo interpartal ocasionando baixo peso nos recém-nascidos ${ }^{(3)}$.

Mulheres que iniciam a maternidade na adolescência, tendem a ter um número maior de filhos durante toda a sua vida reprodutiva. $\mathrm{Na}$ maioria dos casos, a primeira gravidez não é planejada, e algumas vezes indesejada. Assim, a probabilidade das seguintes gestações adquirirem o caráter não desejado da primeira torna-se altíssima.

Considerando o desenvolvimento tecnológico que hoje presenciamos no campo da contracepção e os avanços no âmbito da saúde sexual e reprodutiva, disponibilizar informações e meios relacionados aos métodos anticoncepcionais existentes é uma das melhores formas de adesão a um programa de prevenção. Oferecer opções de escolha à jovem, ou até mesmo ao casal, gera segurança e, conseqüentemente, melhor utilização do método. Este é um momento importantíssimo, implica em uma vida sexual segura e satisfatória, tendo a capacidade de reproduzir e a liberdade de decidir sobre quando e quantas vezes deve fazê-lo.

Diante da relevância do tema e na constatação do elevado número de repetição de gravidezes entre adolescentes em nosso meio e, ainda, considerando a problemática do seu não planejamento com repercussões na formação acadêmica e profissional dos jovens, faz-se necessária a mobilização da sociedade por meio de programas de saúde, possibilitando a este grupo da população maior acesso a informações e meios que lhes permitam desenvolver e praticar uma postura crítica, consciente e responsável no exercício da sua sexualidade.

Neste contexto, este estudo teve como objetivos:

- Identificar o perfil epidemiológico de adolescentes que tiveram pelo menos uma gravidez antes de matricularem-se em um programa de planejamento familiar.

- Avaliar os efeitos de um programa educativo e assistencial de planejamento familiar frente a reincidência de gestação em adolescentes.

\section{MÉTODOS}

Trata-se de um estudo descritivo e retrospectivo realizado no setor de Planejamento Familiar na Universidade Federal de São Paulo - UNIFESP/EPM que, desde 1992, inclui um serviço específico de atendimento e atividades de assistência à população de adolescentes.

A amostra populacional foi composta por prontuários de adolescentes do sexo feminino, com idade entre $10 \mathrm{e}$ 19 anos completos, matriculadas no Programa de Planejamento Familiar, no período de janeiro de 1998 a dezembro de 2003. Como critério de inclusão da amostra, foi utilizada a ocorrência de, no mínimo, uma gestação anterior à data da matrícula no programa. Ressalta-se que a pesquisa foi realizada com o consentimento dos profissionais responsáveis pelo programa, e também com a aprovação do Comitê de Ética em Pesquisa da Universidade.

O número total de adolescentes matriculadas no período foi de 583 considerando os critérios de inclusão, a amostra foi constituída por 264 prontuários, que representaram $45,3 \%$ deste total no período estudado de cinco anos estudado. Para a coleta dos dados foi utilizado um formulário estruturado, de forma a facilitar e organizar a coleta e a análise dos prontuários.

As variáveis estudadas foram:

- Sócio-demográficas - Idade, situação conjugal, escolaridade e tempo de matrícula no serviço;

- Obstétricas e ginecológicas - idade: da menarca; do início da atividade sexual; da primeira gravidez; número de gestações, paridade e abortos antes da matricula no serviço; número e razão da(s) gravidez(es) após matrícula no serviço e intervalo(s) intergestacional(is);

- Anticonceptivas - métodos contraceptivos e tempo de uso antes da gravidez e métodos contraceptivos e tempo de uso após a matrícula no serviço.

\section{RESULTADOS E DISCUSSÃO}

A adolescência é um período da vida em que ocorre um importante desenvolvimento que torna o indivíduo apto a perpetuação e reprodução da espécie. Como o desenvolvimento psíquico ocorre após o orgânico, a menina pode chegar aos ciclos ovulatórios que indicam a maturidade orgânica para a reprodução, passando a apresentar corpo de mulher jovem e fértil, sem ainda ter amadurecimento emocional para administrá-lo.

Deve-se considerar que a média de idade da menarca vem apresentando uma tendência de queda, diminuindo cerca de quatro meses a cada década, encontrando-se 
atualmente na faixa dos 11 a 12 anos. Trabalhos nacionais relatam que na década de 1930 encontrava-se por volta dos 13,6 anos, 13,4 anos na década de 1940; 12,8 anos, na década de 1960 e 12,6 anos na de $1980^{(4)}$.

Aliada a grande oportunidade para manter relações sexuais, devido ao estilo de vida moderno e aos estímulos ambientais, cada vez maiores, a iniciação sexual tem-se mostrado ocorrer de forma mais precoce. Como conseqüência, depara-se freqüentemente com situações de risco, como a gravidez não planejada e indesejada.

Nossos resultados revelam que a amplitude de variação na idade das adolescentes foi de 14 a 19 anos, com média de 17,5 anos. Ao matricularem-se no programa, cerca de $50 \%$ viviam junto com o companheiro, não possuindo uma união estável por lei, 31\% eram casadas e 1,5\% já viúvas, fruto, provavelmente, da violência urbana que hoje vivemos.

Em relação à escolaridade, o estudo mostra 11,0\% das jovens com pouco aproveitamento escolar sendo 4,5\% analfabetas e 6,5\% terem, até o momento da matrícula no programa, de um a quatro anos de estudo. No entanto, nota-se expectativa positiva em $89,0 \%$ da amostra, com cinco a 12 anos de estudo, média de 7,8 anos. Este resultado de certa forma nos surpreendeu, uma vez que a gravidez precoce, e muitas vezes não desejada, vulnerabiliza adolescentes jovens, principalmente mulheres, roubando-lhes a oportunidade de pleno desenvolvimento a que têm direito, favorecendo menor chance de crescimento pessoal e profissional.

Pesquisa realizada pelo Fundo das Nações Unidas para a Infância aponta que em 2001/2002, 94\% dos adolescentes brasileiros entre 12 e 17 anos achavam-se matriculados em algum estabelecimento de ensino, e destes, $46 \%$ eram do sexo feminino ${ }^{(5)}$. Ao se levar em conta o local de residência, esta situação inverteu-se, elevando-se para 53,3\% o índice de jovens residentes nas capitais, matriculadas.

Em estudo anterior realizado no Brasil pela Organização Mundial da Saúde com 157 mães adolescentes foi encontrado que a gravidez pode determinar a interrupção do processo formal de educação das jovens, pois $97,1 \%$ das pesquisadas tinham nove anos ou menos de escolaridade e já se encontravam fora da escola quando do início da gestação ${ }^{(6)}$. Os resultados obtidos em nosso estudo, revelam semelhanças neste aspecto.

A fecundidade tende a diminuir com o aumento da escolaridade e do nível de rendimento. Este é um indicador que deve ser incorporado pelos gestores de políticas públicas. O incremento da educação no país e o incentivo para que os jovens prossigam na educação formal, além do ensino fundamental, apresenta reflexos imediatos na saúde sexual e reprodutiva da população.

Nas últimas décadas, vários estudos vêm demonstrando que jovens de ambos os sexos iniciam relações sexuais precocemente. Em 1996, os rapazes tinham a primeira relação sexual mais cedo do que as moças, sendo a mediana da idade 15 anos para os homens e 16 para as mulheres ${ }^{(7)}$.

Os dados obtidos no presente estudo confirmam que além da menarca estar sendo antecipada, o início da atividade sexual também está ocorrendo de forma precoce, isto é, em média por volta dos 15 anos. Nossos achados encontram-se em concordância com os de outros estudiosos no assunto, ao relatarem que a faixa etária mais freqüente para o início das relações sexuais está entre os 15 ou 16 anos $^{(8)}$. Estes índices podem ser explicados pelo estilo de vida moderno bem como pelos fatores do meio ambiente e da mídia a que os jovens estão expostos.

Em 1999 o Brasil possuía 23\% de mães menores de 20 anos de idade. Em 2000, do total de adolescentes, na faixa etária de 10 a 14 anos, 0,43\% tinha filhos e $17 \%$ delas, mais de um filho. Ainda que essa faixa etária não apresente significativo impacto sobre a taxa de fecundidade no período de 1996 a 2000 houve um acréscimo de $1,8 \%$ no percentual de partos na faixa etária dos 10 aos $14 \operatorname{anos}^{(9)}$.

Nosso estudo revela que ao matricularem-se no programa de planejamento familiar, $73,5 \%$ já possuíam uma gestação, $24,2 \%$ duas e $2,3 \%$ três. Deste total de gestações, $11 \%$ abortaram e $89 \%$ tiveram, em média, 1,4 filhos. As jovens engravidaram cerca de um ano após o início da vida sexual ou no seu decorrer, uma vez que, em média, a primeira relação ocorre aos 15 anos e a primeira gestação aos 16,1 anos. Este fato pode ser provavelmente explicado pelo desconhecimento da forma segura de prevenir estas situações ou do uso correto do método e, mais ainda, pelos aspectos importantes das manifestações de sua sexualidade.

A antecipação do início da atividade sexual entre os jovens faz com que muitas das adolescentes procurem os métodos contraceptivos como forma de evitar uma gravidez não desejada. Porém, há uma notável tendência de opção pelo condom, em especial nas situações onde não se tem certeza da monogamia de um dos parceiros ou de ambos, aliando-se, a outro método de maior eficácia, quando a anticoncepção for por adolescentes.

Tabela 1- Métodos contraceptivos utilizados, antes e após matrícula no programa de planejamento familiar. São Paulo, 2004.

\begin{tabular}{l|cc}
\hline \multirow{1}{*}{ Método } & Antes & Após \\
\cline { 2 - 3 } & $\mathbf{n}$ & $\mathbf{n}^{*}$ \\
\hline Condom & 105 & 224 \\
Hormonal oral & 106 & 102 \\
Hormonal injetável & 23 & 75 \\
Nenhum/Ignorado & 28 & 52 \\
DIU & - & 2 \\
Outro & 2 & 2 \\
\hline
\end{tabular}

* Uso isolado ou associado 
A partir dos resultados encontrados, pode-se confirmar a parceria de utilização de métodos contraceptivos, tendo como exemplo a pílula e o condom. Talvez esteja havendo maior conscientização por parte das jovens em relação à dupla proteção, prevenindo as DSTs e a gravidez. Este pensamento pode ser confirmado pelo baixo número de jovens (28) encontrado que antes de matricularem-se no programa, não utilizavam nenhum método contraceptivo e após a matrícula foi duplicada a opção pelo uso do condom, quer seja de forma isolada ou combinada ou pelo método anticoncepcional hormonal. Acredita-se que este achado está relacionado com a ênfase dada pelos profissionais do serviço à dupla proteção, pela oferta do método e fácil acesso. Ressaltase, ainda que cerca de $70 \%$ das adolescentes utilizam os métodos por, no máximo, seis meses. Reforçando esta idéia, estudos semelhantes revelam que as jovens que consideram ter um relacionamento estável (maior que seis meses), abandonaram os métodos utilizados ${ }^{(10)}$. Este ponto é ainda reforçado pela recusa do uso do preservativo pelo parceiro.

Ao compararmos os resultados, podemos afirmar que mesmo após a inserção em um programa de educação sexual e reprodutiva, os métodos mais utilizados e de maior preferência pelas jovens, continuam sendo o condom e o anticoncepcional hormonal oral. Entre os que foram classificados como métodos ignorados encontram-se as jovens que somente compareceram ao programa de planejamento familiar uma única vez, no dia da matrícula. Tendo por base somente o dado de uma consulta, torna-se impossível relatar a utilização ou não de algum método contraceptivo pelas mesmas.

Tabela 2- Gravidez e razão de sua ocorrência após matrícula no programa de planejamento familiar. São Paulo, 2004.

\begin{tabular}{|c|c|c|}
\hline Gravidez & $\mathbf{n}$ & $\%$ \\
\hline Nenhuma & 251 & 95,1 \\
\hline$\underline{1}$ & 13 & 4,9 \\
\hline Total & 264 & 100,0 \\
\hline Razão & $\mathbf{n}$ & $\%$ \\
\hline Desejada pela adolescente & 1 & 7,7 \\
\hline Desejada pelo casal & 1 & 7,7 \\
\hline Falha do método & 1 & 7,7 \\
\hline Utilização errada do método & 10 & 76,9 \\
\hline Total & 13 & 100,0 \\
\hline
\end{tabular}

Em relação a reincidência gestacional, encontramos resultado satisfatório do programa de planejamento familiar pesquisado, apresentando taxa de $4,9 \%$ entre as adolescentes matriculadas (Tabela 2). Mesmo considerando um significante número de abandono do programa, a taxa continua sendo muito favorável.
Observa-se, também, que na amostra pesquisada, apenas 13 adolescentes engravidaram novamente.

Ao compararmos este dado com outro que faz referência ao mesmo programa de planejamento familiar para adolescentes, nota-se um aumento de $11,8 \%$ da taxa de aproveitamento do serviço (ausência de repetição de gravidez) ${ }^{(11)}$. Ainda dentro destas observações, encontrou-se apenas uma jovem que teve duas gravidezes reincidentes após inserção no programa. Dentre as razões apontadas para a ocorrência da gravidez, 50\% referem que engravidaram por abandono do método ou má utilização deste. Estes dados expostos, comparados com o presente estudo, referem que apenas $15,4 \%$ das reincidências das gravidezes foram desejadas, sendo que $7,7 \%$ (um caso) ocorreu por falha do método (DIU). A grande porcentagem encontrada de novas gravidezes $(76,9 \%)$ foi decorrente da utilização errada do método (Tabela 2).

Tabela 3- Intervalo, em meses, entre gestações em adolescentes matrículadas no programa de planejamento familiar. São Paulo, 2004.

\begin{tabular}{lcc}
\hline \multicolumn{1}{c}{ Intervalo entre a $\mathbf{1}^{\mathbf{a}}$ e a $\mathbf{2}^{\mathbf{a}}$ gestação } & $\mathbf{n}$ & $\mathbf{0}$ \\
\hline 1 a 6 & 10 & 14,9 \\
7 a 12 & 24 & 35,8 \\
13 a 18 & 17 & 25,4 \\
19 a 24 & 12 & 17,9 \\
? 25 & 4 & 6,0 \\
\hline Média & 13,3 & \\
\hline Total & $\mathbf{6 7}$ & $\mathbf{1 0 0 , 0}$ \\
\hline \hline Intervalo entre a $\mathbf{2}^{\mathbf{a}}$ e a $\mathbf{3}^{\mathbf{a}}$ gestação & $\mathbf{n}$ & $\mathbf{\%}$ \\
\hline \hline 6 & 1 & 20,0 \\
9 & 1 & 20,0 \\
14 & 1 & 20,0 \\
20 & 1 & 20,0 \\
22 & 1 & 20,0 \\
\hline Média & 14,2 & \\
\hline Total & $\mathbf{5}$ & $\mathbf{1 0 0 , 0}$ \\
\hline
\end{tabular}

Os resultados apontam um número significativo de adolescentes com intervalo interpartal da primeira para a segunda gravidez entre sete e 12 meses, representando cerca de 36\% da amostra (Tabela 3 ).

O mesmo intervalo foi obtido em estudo realizado com 26 adolescentes grávidas na cidade de Ribeirão Preto/SP ${ }^{(12)}$. Este intervalo é considerado reduzido para a recuperação das condições que o organismo feminino necessita para um desenvolvimento obstétrico seguro, especialmente na adolescente. Reiterando os resultados obtidos, apenas cinco das adolescentes estudadas tiveram uma terceira gravidez, sendo que duas delas ocorreram no período de matrícula no serviço. A média, em meses, 
de intervalo entre a segunda e a terceira gestação foi de 14,2, ou seja, aproximadamente um ano, confirmando os dados acima analisados.

\section{CONCLUSÕES}

Conclui-se que as jovens, ao matricularem-se no programa de planejamento familiar, na sua maioria já vivenciaram pelo menos uma gestação, tendo esta evoluído ou não para parto. Esta gravidez ocorre cerca de um ano após a sexarca e, em média, aos 16 anos. A instabilidade emocional, bem como o relacionamento com o sexo oposto, faz com que elas utilizem por, no máximo seis meses, um método contraceptivo, antes de matricularem-se em um programa de planejamento familiar; sendo os de maior prevalência o condom e o anticoncepcional hormonal oral. A aderência ao programa acontece, em média, por 12 meses, onde os métodos de maior escolha encontram-se entre o condom e os anticoncepcionais hormonais. A reincidência de gravidez apresenta índice de $4,9 \%$ entre todas as jovens matriculadas e que deram continuidade ao programa. Destas, 15,4\% foram desejadas sendo que em 7,7\% ocorreu a falha do método. A grande porcentagem encontrada de novas gravidezes, 76,9\%, foi decorrente da utilização incorreta do método.

Os dados encontrados no presente estudo permitem reconhecer a vulnerabilidade e a exposição das adolescentes à gravidez e sua repetição. Reforçam a importância do estabelecimento de políticas públicas e programas voltados para a saúde sexual e reprodutiva dos adolescentes e jovens que englobem a educação, os conceitos e o uso correto dos métodos contraceptivos, que ofereçam além do método, o acompanhamento médico e de enfermagem, visto a necessidade destes de informações e meios de prevenção de gravidez.

\section{REFERÊNCIAS}

1. Rede Feminista de Saúde. Adolescentes saúde sexual saúde reprodutiva: dossiê. Belo Horizonte; 2004.

2. Brasil, Ministério da Saúde. Instituto Nacional de Alimentação e Nutrição. Coordenação da Saúde da Mulher, do Adolescente e da Criança, COSAM. Pesquisa Nacional sobre Demografia e Saúde, 1996. Rio de Janeiro, 1997.

3. Cockey CD. Preventing teen pregnancy. It's time to stop kidding around. AWHONN Lifelines. 1997; 1(3):32-40.

4. Guazzelli CAF, Lindsey PC, Aldrighi JM, Petta CA. Anticoncepção na adolescência. In: Aldrighi JM, Petta CA, editors. Anticoncepção: aspectos contemporâneos.São Paulo: Atheneu; 2005. p.129-34.

5. Fundo das Nações Unidas para a Infância. UNICEF. A voz dos adolescentes. Brasília; 2002.

6. Silva JLP, Nogueira CWM. A multigravidez na adolescência. In: Organização Panamericana da Saúde e OMS. Coletânea sobre saúde reprodutiva do adolescente brasileiro. Brasília : Organização Panamericana da Saúde/OMS; 1988. p.101-11.

7. Brasil Ministério da Saúde, Secretaria de Políticas de Saúde, Coordenação Nacional de DST e Aids. Comportamento sexual da população brasileira e percepções do HIV/AIDS. Brasília: A Coordenação; 2000

8. Vitiello N. Planejamento familiar para adolescentes. Reprodução. 1991; 6(4):159-68.

9. Brasil. Ministério da Saúde. Secretaria de Atenção à Saúde. Departamento de Ações Programáticas Estratégicas. Área Técnica de Saúde da Mulher. 'Política nacional de atenção integral à saúde da mulher - princípios e diretrizes”. Brasília; 2004.

10. Olinto MTA, Galvão LW. Características reprodutivas de mulheres de 15 a 49 anos: estudos comparativos e planejamento de ações. Rev Saúde Pública = J Public Health. 1999; 33(1):64-72.

11. Lopes DM, Barbieri M, Guazzelli CAF. Efeitos de um Programa em Planejamento Familiar na vida reprodutiva de adolescentes pós-ocorrência de abortamento. Acta Paul Enfermagem. 2001;14(2):38-45.

12. Persona L, Shimo AKK, Tarallo MC. Perfil de adolescentes com repetição da gravidez atendidas num ambulatório de pré-natal. Rev Latinoam Enfermagem. 2004; 12 (5):745-50. 